\title{
Spin-Dependent Emission from Arrays of Planar Chiral Nanoantennas Due to Lattice and Localized Plasmon Resonances
}

\author{
Michele Cotrufo, ${ }^{* \dagger, \ddagger}$ Clara I. Osorio, ${ }^{\ddagger}$ and A. Femius Koenderink ${ }^{\ddagger}$ \\ ${ }^{\dagger}$ COBRA Research Institute, Eindhoven University of Technology, 5600 MB Eindhoven, The Netherlands \\ ${ }^{\ddagger}$ Center for Nanophotonics, FOM Institute AMOLF, Science Park 104, 1098 XG Amsterdam, The Netherlands
}

Supporting Information

\begin{abstract}
Chiral plasmonic nanoantennas manifest a strong asymmetric response to circularly polarized light. Particularly, the geometric handedness of a plasmonic structure can alter the circular polarization state of light emitted from nearby sources, leading to a spin-dependent emission direction. In past experiments, these effects have been attributed entirely to the localized plasmonic resonances of single antennas. In this work, we demonstrate that, when chiral nanoparticles are arranged in diffractive arrays, lattice resonances play a primary role in determining the spindependent emission of light. We fabricate $2 \mathrm{D}$ diffractive arrays of planar chiral metallic nanoparticles embedded in a lightemitting dye-doped slab. By measuring the polarized photo-

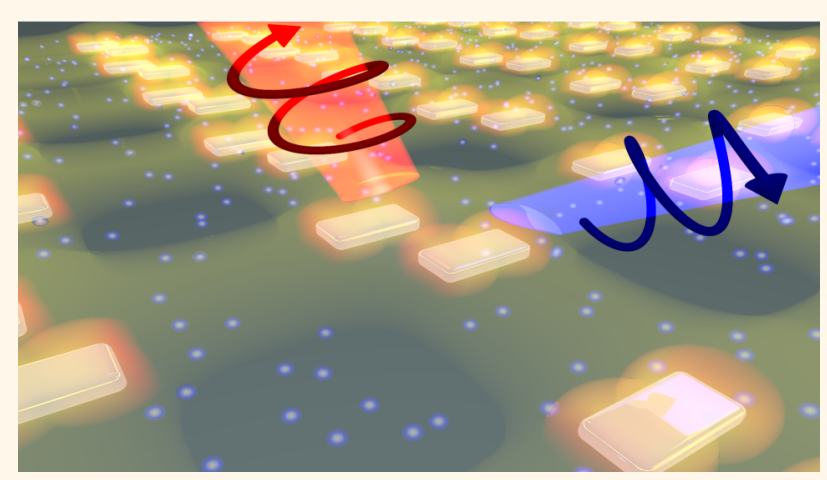
luminescence enhancement, we show that the geometric chirality of the array's unit cell induces a preferential circular polarization, and that both the localized surface plasmon resonance and the delocalized hybrid plasmonic-photonic mode contribute to this phenomenon. By further mapping the angle-resolved degree of circular polarization, we demonstrate that strong chiral dissymmetries are mainly localized at the narrow emission directions of the surface lattice resonances. We validate these results against a coupled dipole model calculation, which correctly reproduces the main features. Our findings demonstrate that, in diffractive arrays, lattice resonances play a primary role into the light spin-orbit effect, introducing a highly nontrivial behavior in the angular spectra.
\end{abstract}

KEYWORDS: localized plasmon resonances, surface lattice resonances, $k$-space polarimetry, chirality, plasmonic nanoantennas, spin-orbit

$\mathrm{P}$ lasmonic nanoantennas can control, manipulate, and redirect emission of light by providing an interface between plane waves in the far-field and localized emitters in the near-field. ${ }^{1}$ While intrinsically based on the material resonance of free electrons, shape and aspect ratio of these nanoantennas can be used to tune antenna performance in terms of resonance frequency, near-field enhancement, and spontaneous emission decay rate enhancement. ${ }^{2}$ Moreover, antenna shape can impart preferential polarization on the emission of nearby emitters. ${ }^{2}$ Indeed, localized surface plasmon resonances (LSPRs) supported by anisotropic structures such as nanorods, bowtie antennas, patch antennas, or split rings can strongly favor particular electric field components. ${ }^{3,4}$ Therefore, light polarization plays a key role in the behavior of nanoantennas.

Strong polarization responses are not exclusive to linear polarization: plasmonic antennas can be highly selective with respect to the helicity of light if they possess a geometric chirality. ${ }^{5}$ Chiral objects cannot be superimposed onto their mirror image by mere rotations and translations and interact differently with right-handed (RCP) and left-handed (LCP) circularly polarized light (CPL). For chiral molecular matter, this interaction is usually described in terms of far-field quantities like optical activity, which describes the rotation of incident linear polarization, or circular dichroism, which accounts for preferential absorption of right- or left-handed circularly polarized light. Several experimental and theoretical studies have shown that planar chiral structures such as gammadions $^{6}$ or spirals ${ }^{7}$ and 3D chiral structures like helices ${ }^{8,9}$ can exhibit large optical activity and circular dichroism ${ }^{10-14}$ and can enhance the weak circular dichroism signals obtained from biological chiral molecules. ${ }^{5,15}$ Chiral nanostructures can also

Received: November 16, 2015

Accepted: February 8, 2016

Published: February 8, 2016 

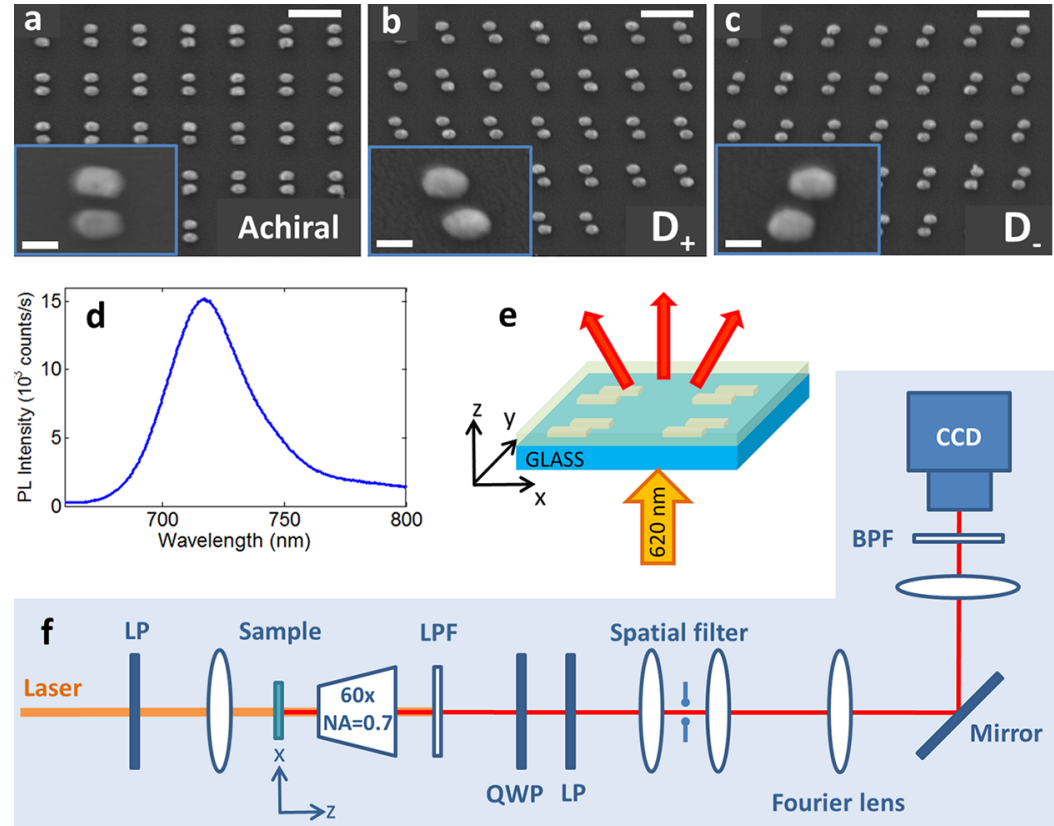

Figure 1. (a-c) SEM micrographs of the arrays of (a) achiral, (b) $D_{+}$, and (c) $D_{-}$structures. The pitch of the array is $480 \mathrm{~nm}$. The scale bars of the main pictures are $500 \mathrm{~nm}$. The inset of each figure shows a zoomed image on the corresponding unit cell. The scale bar is $100 \mathrm{~nm}$. (d) Fluorescence emission from the dye Rh800 collected from a region far from the plasmonic structures. (e) Schematic of the sample, the experiment configuration for the $k$-space polarimetry measurements, and the reference frame. (f) Schematic of the setup. The laser light $(\lambda=$ $620 \mathrm{~nm}$ ) is focused on the sample from the glass side, and the luminescence is collected on the other side through a $60 \times$ objective. After filtering out the laser with a long-pass filter (LPF) and selecting the desired state of polarization with the quarter wave plate (QWP) and the linear polarizer (LP), the beam is spatially filtered with a $400 \mu \mathrm{m}$ diameter pinhole. A flippable Fourier lens allows the CCD to image either the plane of the sample or the back focal plane of the objective. A band-pass filter (BPF) in front of the camera allows monochromatic Fourier images to be acquired.

alter the far-field polarization state of fluorescent sources positioned in their near-field. Split-ring resonators, for example, have been shown to provide spin control ${ }^{16}$ of photons emitted from II-VI semiconductor quantum dots. In a recent experiment by Meinzer et al., ${ }^{17}$ it was shown that a planar chiral arrangement of two silver nanorods embedded in a slab of achiral emitters gives rise to a substantial asymmetry in the intensity of LCP and RCP light emitted normal to the sample. In this experiment, the handedness of emission is inherited from the handed nature of the localized plasmonic resonances of the antennas.

In this work, we investigate periodic arrays of planar chiral antennas interacting with achiral emitters and show that diffractive resonances play a dominant role in determining the spin-dependent directional emission. Plasmonic antenna arrays with pitches comparable to the wavelength are known to exhibit hybrid plasmonic-photonic modes with very narrow line widths ${ }^{18}$ known as surface lattice resonances (SLRs). ${ }^{19}$ These modes arise from radiative coupling between LSPRs and waves diffracted into the plane of the array. For simple antenna shapes, like rods, this hybrid resonance has been shown to strongly improve the photoluminescence properties of nearby emitters $^{20}$ and, in particular, to drastically modify their angular emission pattern. ${ }^{21}$ While Meinzer et al. ${ }^{17}$ and Kruk et al. ${ }^{16}$ attributed chiral emission properties to the physics of single antennas, their structures were actually arranged in arrays, triggering the question of how surface lattice resonances impact handedness in emission.

We report $k$-space polarimetry measurements ${ }^{22,23}$ that quantify how asymmetries in circularly polarized light emission are distributed over an angle, as mapped across the back aperture of a microscope objective. We demonstrate that the SLR introduces much larger CPL asymmetries at selected angles than what is observed in experiments that only consider emission normal to the array.

Sample Design, Optimization, and Characterization. In this work, we study periodic arrays of chiral and achiral dimer antennas fabricated on a glass substrate and embedded in a thin dye-doped polymer layer. We emphasize that, apart from the small refractive index contrast introduced by the substrate, the structures considered in this work are not 3D chiral, but they possess a planar chirality. Previous works have shown ${ }^{24}$ that planar chiral structures feature strong asymmetric response for the transmission of CPL because the handedness is reversed when they are observed from opposite sides.

The achiral dimers consist of two identical silver rod antennas shifted along their short dimension (Figure 1a). The planar chiral structures were obtained by further shifting the rods along their long dimension to obtain a relative displacement equal to half their length (i.e., $60 \mathrm{~nm}$ in the geometry we adopted; see below). According to the direction of the shift, we labeled the dimers $D_{+}$and $D_{-}$, as shown in the inset of Figure $1 b, c$. This unit cell is based on the design by Meinzer et al. ${ }^{17}$ Figure 1e illustrates schematically the geometry of the sample, the chosen reference frame, and the excitationcollection configuration used for the $k$-space polarimetry measurements discussed below.

The geometry of the sample (rods length and array pitch) was optimized using finite-difference time domain (FDTD) simulations (with a commercial solver ${ }^{25}$ ) to ensure that both the LSPR and the SLR wavelength fall in the emission band of the dye used in this experiment, that is, $\lambda \approx 700-780 \mathrm{~nm}$ (see 
Figure 1d). We notice that further optimizations (e.g., the gap between the rods) could lead to larger values of the chiral dissymmetry, but this would go beyond the scope of the present work. We calculated normal-incidence transmittance spectra of the sample for different nanorod lengths, array pitches, and impinging polarizations. Figure 2a,b shows the
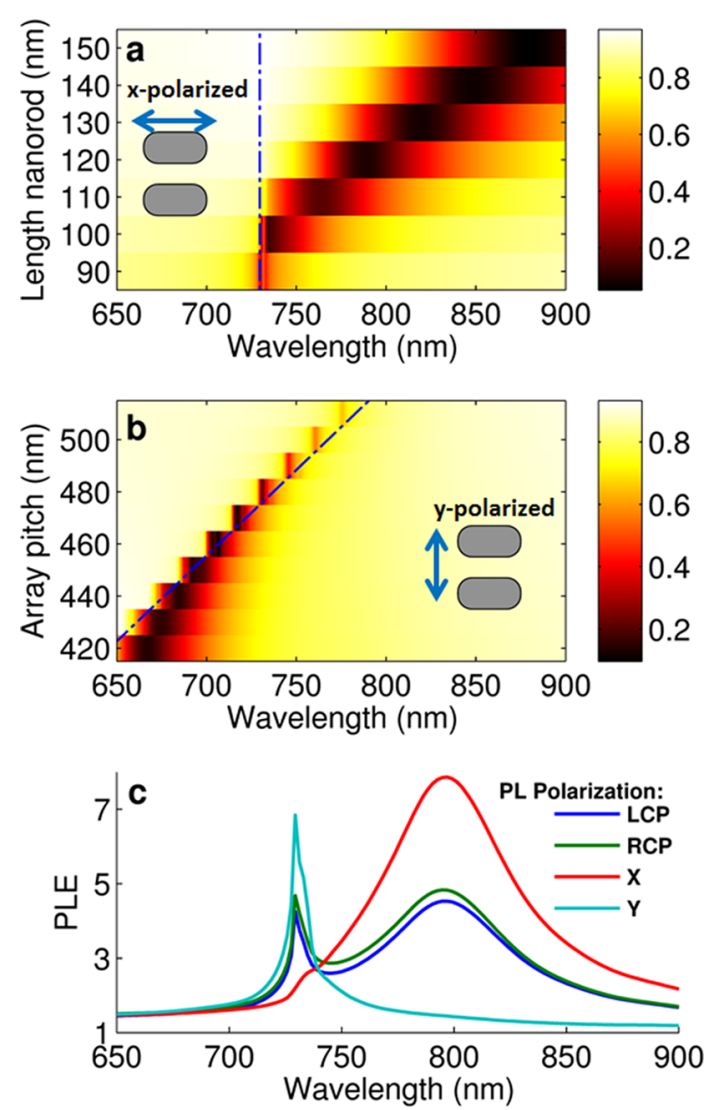

Figure 2. Numerical optimization of the sample geometry. (a) Calculated $x$-polarized transmittance of an array of achiral structures for different lengths $L$ of the rods and for an array pitch of $p=480 \mathrm{~nm}$. The vertical dashed-dotted line shows the position of the Rayleigh's anomaly. (b) Calculated $y$-polarized transmittance of an array of achiral structures for different array pitches $p$ and for a rod length of $120 \mathrm{~nm}$. The dashed-dotted line shows the dispersion relation of the Rayleigh's anomaly. (c) Calculated luminescence enhancement for a $D_{+}$structure with $L=$ $120 \mathrm{~nm}$ and $p=480 \mathrm{~nm}$ for different luminescence polarizations.

different behaviors for light linearly polarized along (panel a) and perpendicular to (panel $b$ ) the dimer rods. The transmittance of an $x$-polarized beam (Figure 2a) shows a broad minimum, whose spectral position strongly depends on nanorod length. This feature is identified as the dipolar LSPR of the dimer plasmonic antenna. For polarization rotated by $90^{\circ}$, a much narrower transmission minimum appears, with a spectral position strongly affected by the lattice period (Figure $2 \mathrm{~b}$ ) but independent of the nanorod length (see Supporting Information). Moreover, the dip's wavelength lies close to the Rayleigh anomaly condition, ${ }^{18}$ indicated by the dashed-dotted blue line in Figure $2 \mathrm{~b}$ and given by $\lambda=n_{\text {glass }} \times p$, where $n_{\text {glass }}$ is the substrate refractive index and $p$ is the lattice pitch. These characteristics are typical signatures of the SLR ${ }^{18}$ Based on these simulations and on the emission band of the dye used (see Figure 1d), we set the single rod dimensions to $120 \mathrm{~nm} \times$
$70 \mathrm{~nm} \times 30 \mathrm{~nm}$ and the array pitch to $p=480 \mathrm{~nm}$ in our experiments. The vertical shift between the rod centers is 130 $\mathrm{nm}$, and in simulations and experiments the rods are embedded in a $65 \mathrm{~nm}$ thick SU8 polymer layer hosting the dye Rh800.

Having chosen a promising geometry, we evaluated the average of the square modulus of the electric field in the dyedoped polymer layer for different impinging beam polarizations. Due to the reciprocity theorem, this calculation gives a prediction for the far-field polarized luminescence enhancement of the dye (averaged over all possible positions in the polymer slab) expected for emission normal to the sample plane. Figure $2 \mathrm{c}$ shows the calculated photoluminescence enhancement (PLE) for an array of $D_{+}$structures. The predicted $x$-polarized luminescence enhancement shows a broad feature whose position and line width agree well with the $x$-polarized LSPR. The $y$-polarized signal is instead characterized by a narrow peak, due to the SLR. When we consider the right- and left-handed circularly polarized components of the luminescence enhancement, a clear difference between the two signals is visible for both the LSPR and the SLR. In other words, the simulations predict a helicity-dependent photoluminescence enhancement for observation normal to the sample, in agreement with previous observations. ${ }^{17}$

Figure 3a shows measured transmittance spectra of the sample under white light illumination for different impinging polarizations and different chirality of the plasmonic structures. The transmittance of an $x$-polarized (dashed lines) and $y$ polarized (solid lines) excitation beam shows the signature of the LSPR and SLR, respectively, in good agreement with the calculations (Figure 2a,b). The transmittance curves for the achiral and chiral structures are, even for a linearly polarized input, slightly different: the SLR minimum from an array of chiral structures is blue-shifted by about $10 \mathrm{~nm}$ with respect to an array of achiral antennas. For the LSPR, the shift has approximately the same absolute value but is opposite in sign. We attribute this spectral shift to the different near-field interactions between the two silver rods, caused by the different geometry of the achiral and chiral structures.

\section{RESULTS AND DISCUSSION}

Chiral Photoluminescence Enhancement from SRL and LSPR. We start by studying how the LSPR and SLR modify the emission spectra of the dye, measuring in a low-NA geometry similar to the configuration used by Meinzer et al. ${ }^{17}$ In these measurements, the sample is pumped from the polymer side and the luminescence is collected from the glass side. To calculate the polarized photoluminescence enhancement, we measured the LCP, RCP, and $x$ - and $y$-polarized components of the photoluminescence. Each measurement was normalized to a reference measurement (with the same polarization) taken from a region of the sample without structures. Figure $3 \mathrm{~b}-\mathrm{d}$ shows the results for the achiral, $D_{+}$, and $D_{-}$structures. For the array of achiral structures, the polarized PLE shows two distinct features for the $x$ (red line) and $y$ (cyan line) polarizations (Figure $3 b$ ). The wavelengths and line widths of these features agree very well with the transmittance measurements for the corresponding excitation polarizations. Therefore, we interpret these enhancements as the result of the interaction between the emitters and the LSPR and SLR of the particles' array. Circularly polarized PLEs show no difference between LCP and RCP light for the achiral structures (blue and green curves in Figure $3 b$ ). 

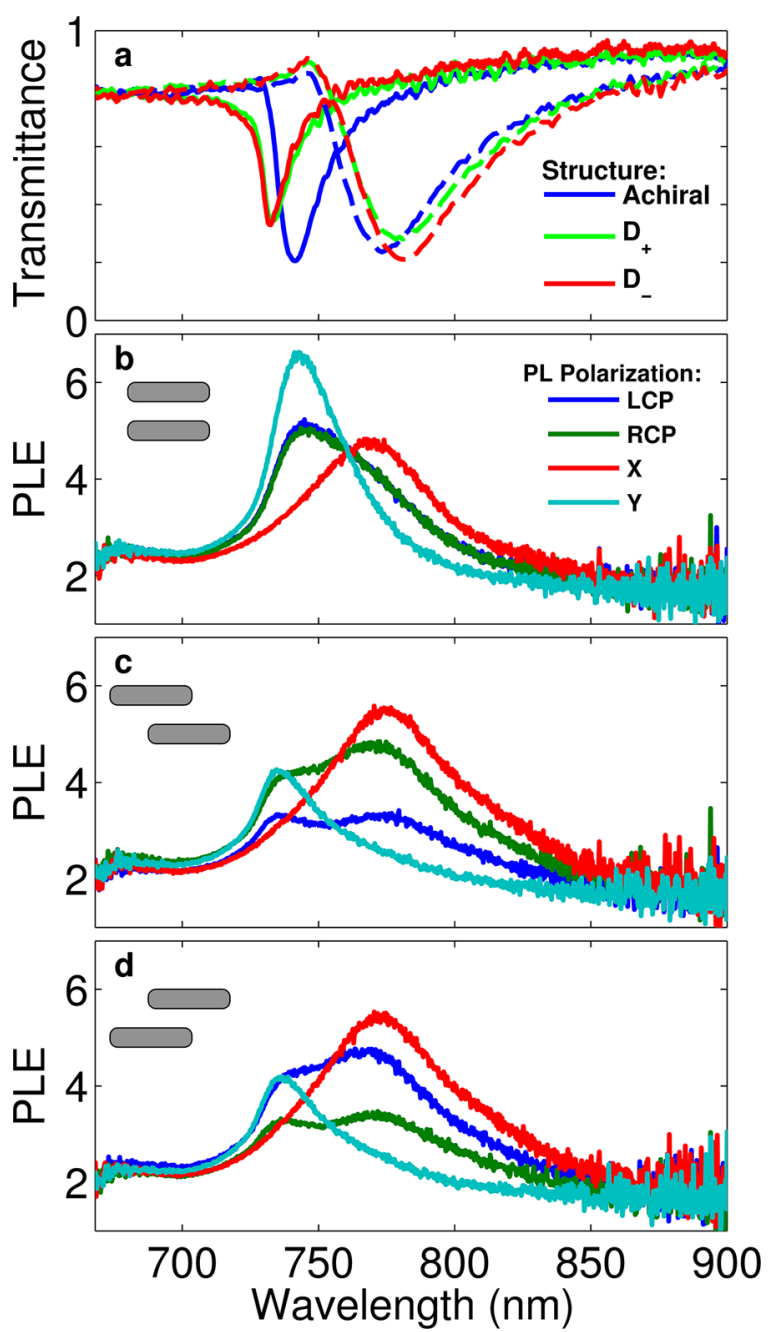

Figure 3. (a) Transmittance spectra of the sample for $y$ - (solid lines) and $x$-polarized (dashed lines) white light illumination and for different chirality of the array's unit cell, as indicated in the legend. (b-d) PLE for different collection polarizations and for (b) achiral, (c) $D_{+}$, and (d) $D_{-}$structures. The geometry of the unit cell is shown in the inset of each plot. The PLE is dominated by the LSPR (SLR) for the $x$-polarized ( $y$-polarized) collection. The LCP and RCP components of the PLE are equal for the achiral structure. A $D_{+}$structure, instead, shows a different PLE for the two circular polarizations; the effect is mirrored for the case of the $D_{-}$structure. In these measurements, the sample is excited from the polymer side and the signal is collected from the glass side.

In contrast to the achiral structures, the chiral structures show a clear difference between the LCP and RCP polarized photoluminescence enhancement. In the wavelength range spanned by the SLR and the LSPR, an asymmetry of about $20 \%$ is observed between the two circular polarizations. This difference is mirrored when inverting the geometric chirality of the structure (cf. Figure $3 \mathrm{c}, \mathrm{d}$ ). In addition to confirming the effect of the LSPR, already reported by Meinzer et al., ${ }^{17}$ we demonstrate here that the emission from the SLR also shows a pronounced asymmetry in the circular polarization of the light emitted. While the measured values of the PLE are in good agreement with the calculations (Figure 2c) for the LSPR, the measurements show a broadening and a weakening of the signal due to the SLR. This effect can be attributed to both fabrication imperfections and to the finite angular acceptance of our setup. As the emission from the SLR strongly changes in angle with wavelength, ${ }^{21}$ the sharp peak calculated for emission normal to the sample (Figure $2 \mathrm{c}$ ) is broadened by angular integration over our setup NA.

Even far from any plasmonic resonances, the measured polarized photoluminescence enhancement has a value of about 2. According to the FDTD calculations (i.e., at fixed source strength), the PLE is expected to be about 1.5 at a wavelength of $650 \mathrm{~nm}$ (see Figure 2c) when ignoring the pump field enhancement. Numerically evaluating the enhancement of the electric field at the laser wavelength excitation $(\lambda=620 \mathrm{~nm})$, we found a spatially averaged value of 1.4 , which leads to an overall expected PLE of about 2.1 (given by the product of the excitation and emission enhancement), in a good agreement with the measurements.

k-Space Polarimetry Measurements on Planar Chiral Plasmonic Antennas. We now discuss the polarized angular emission of the sample measured with the $k$-space polarimetry setup shown in Figure if and described in more detail in the Methods section. As shown in previous works, ${ }^{16,22,23}$ by combining a Fourier microscope and a polarimeter, we can measure the Stokes parameters (which describe the full polarization state of the emitted light; see the Methods section) for each point of the back focal plane of the objective, that is, $S_{i}=S_{i}\left(k_{x}, k_{y}\right)$. Furthermore, by using different narrow band-pass filters in front of the detector, we can measure the Stokes parameters as a function of the emission frequency within the emission band of our dye. Therefore, we characterize the light emitted by a source in terms of momentum, energy, and polarization.

For this measurement, the sample was excited from the glass side and photoluminescence was collected from the polymer side. For the achiral structures, Figure $4 a-f$ shows the change of the intensity distribution in the back focal plane (i.e., $S_{0}$, total intensity) as the emission wavelength varies from 700 to 750 $\mathrm{nm}$ in steps of $10 \mathrm{~nm}$. All of the plots are normalized to their maximum, which is typically on the order of $10^{3}$ counts. The emission patterns show narrow circular features whose position strongly depends on emission wavelength. These emission patterns are characteristic of the $\mathrm{SLR}^{21}$ and result from the diffractive coupling of the LSPR of the single antennas. Each circle corresponds to a single frequency cut through the dispersion relation $\omega\left(\mathbf{k}_{\|}\right)$of the SLR, which is repeated at every reciprocal lattice point $\mathbf{G}_{m, n}=(m, n) \frac{2 \pi}{d}$. Each point of a circle represents a wave vector $\mathbf{k}_{\|}$(allowed for that particular frequency) with origin on the corresponding $\mathbf{G}_{m, n}$ point. To verify the role of the SLR, we fitted the radius of curvature of the observed feature, while keeping the center of the circle fixed at the corresponding reciprocal lattice vector, as shown in Figure $4 \mathrm{f}$. The results of this fitting procedure are shown in Figure $4 \mathrm{~g}$. A linear fit of the data gives a value of $n=1.52 \pm$ 0.01 for the effective refractive index of the SLR. This value is identical to the nominal value of the refractive index of the supporting glass substrate, confirming that the SLR is due to diffractive coupling to glass. ${ }^{26}$

The angular positions of the different features shown in Figure $4 \mathrm{a}-\mathrm{f}$ indicate a clear symmetry in $k$-space, as expected since the array is square. However, a strong asymmetry in the intensity is observed: the vertical features (i.e., the circular segments located in the area $k_{x} \simeq 0$ ) are much brighter than the horizontal ones. This effect can be attributed to the anisotropy of the unit cell, which leads to a mainly $x$-polarized dipole moment for the dimer antennas. As the radiation pattern of an 

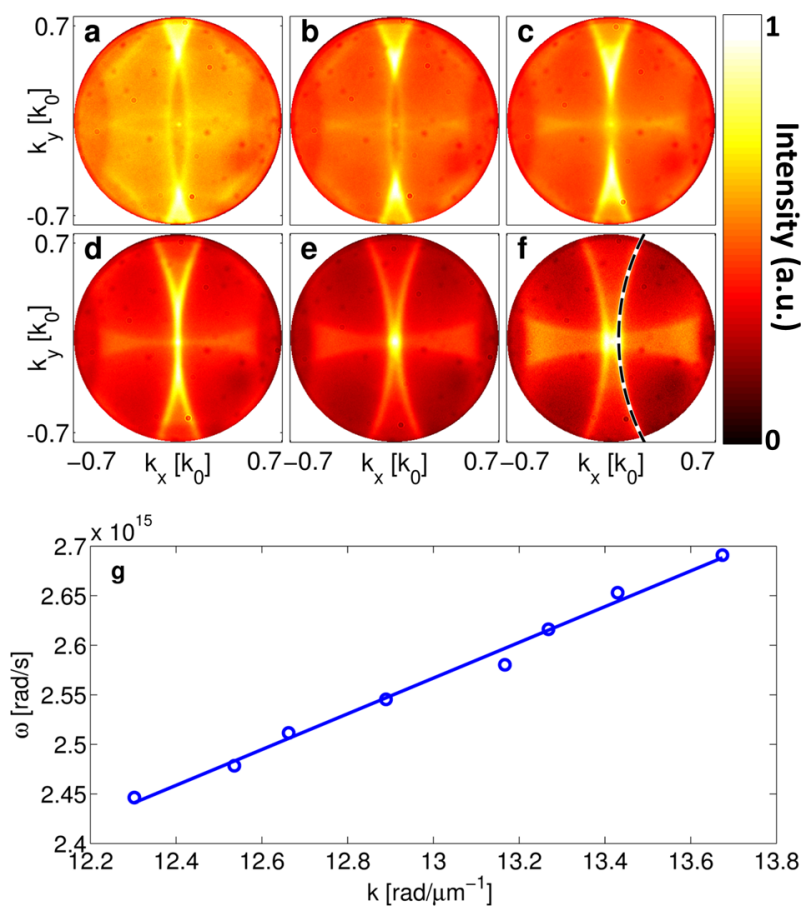

Figure 4. $(a-f)$ Angular distribution of the total emitted intensity (i.e., the Stokes parameter $S_{0}$ ) from the array of achiral structures for different emission wavelengths: (a) $\lambda=700 \mathrm{~nm},(\mathrm{~b}) \lambda=710 \mathrm{~nm}$, (c) $\lambda=720 \mathrm{~nm}$, (d) $\lambda=730 \mathrm{~nm},(\mathrm{e}) \lambda=740 \mathrm{~nm},(\mathrm{f}) \lambda=750 \mathrm{~nm}$. All of the plots are normalized to their respective maximum value. The vertical and horizontal axes indicate the values of the in-plane coordinates of the wave vector, normalized to the absolute value of the corresponding free-space wave vector. The maximum value of 0.7 corresponds to the NA of our objective. The dashed circular segment in (f) shows an example of the fits used to calculate the dispersion curve. (g) Result of the fitting procedure for all the measured wavelengths in the range of $700-770 \mathrm{~nm}$. The solid line is a linear fit to the data, which gives a value of $n=1.52 \pm 0.01$ for the effective refractive index.

electric dipole is maximum at the directions perpendicular to its axis, $^{27}$ a stronger emission in the $y z$ plane is expected, as confirmed by our measurements. For $\lambda=740 \mathrm{~nm}$ (Figure 4e), all of the circles intersect each other at $\left(k_{x}, k_{y}\right) \simeq 0$. This corresponds to a strong emission into the direction orthogonal to the sample plane and coincides with the second-order Bragg diffraction condition for the SLR. Indeed, in the normalincidence transmittance measurements and in the low-NA PLE measurements, the signal related to the SLR appears at $\lambda \simeq 740$ $\mathrm{nm}$.

The sample emission is due to an ensemble of many incoherent and randomly located sources, and therefore, light emitted by different molecules does not interfere. Nevertheless, the patterns observed in Figure $4 \mathrm{a}-\mathrm{f}$ show a clear spatial coherence in the angular spectra. Indeed, as explained by Langguth et al., ${ }^{28}$ summing over the emission of incoherent sources, randomly distributed in a periodic array, still leads to a spatial coherence of emission. The angular spectra of the emission from the ensemble is identical to that of a single source located in a particular position, apart for the smoothing of some intensity variations that occur solely at the crossings of the observed circles. ${ }^{28}$

After having characterized the angle-resolved intensity distribution of emission, we analyzed the angular dependence of the polarization properties, in particular, the degree of circular polarization (DCP, as defined by eq $2 \mathrm{c}$ in the Methods section). Figure $5 \mathrm{a}-\mathrm{e}$ shows the DCP for five emission

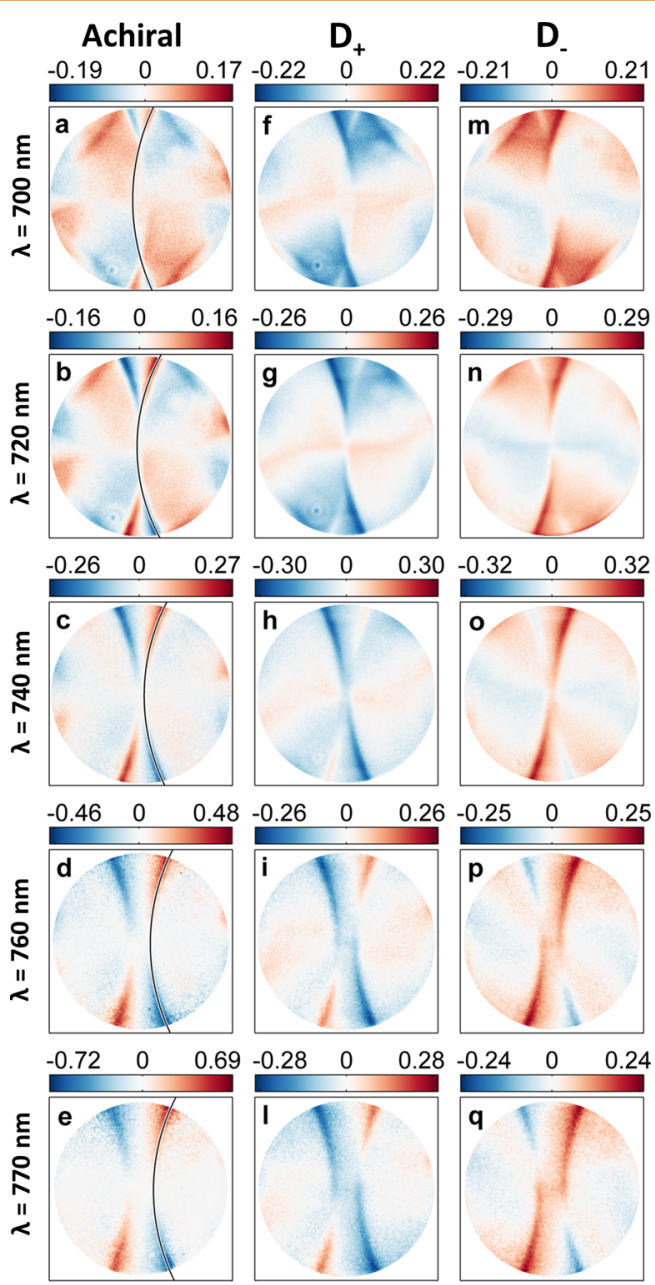

Figure 5. Angle-resolved DCP of the emission from the achiral and chiral structures. The horizontal and vertical axes for each plot are the same as those in Figure $4 a-f$. Each row of the panel corresponds to fixed emission wavelength, ranging from $700 \mathrm{~nm}$ (top row) to $770 \mathrm{~nm}$ (bottom row), as indicated in the label. For each row, the three plots correspond, from left to right, to the emission from the achiral, $D_{+}$, and $D_{-}$structures. The color bar of each plot is indicated on top of it. The black lines in the panels (ae) indicate the SLR emission pattern obtained from the fit of Figure $4 a-f$.

wavelengths $(700,720,740,760$, and $770 \mathrm{~nm})$ for the case of the achiral antennas. According to the definition of DCP, a positive (negative) DCP denotes emission directions for which the RCP (LCP) component of light is more enhanced. Interestingly, even for the achiral geometry, pronounced asymmetries between RCP and LCP (up to a maximum value of $\mathrm{DCP} \simeq 0.7)$ are observed for certain emission angles. The greatest (absolute) values of the DCP are localized along narrow areas which correspond to the SLR emission features. Since in the absence of scatterers the emission is unpolarized for all angles in the objective NA, the induced polarization is due to the LSPR and to the SLR, which is apparent as the set of sharp features. Somewhat similar effects, that is, handed optical responses for nonhanded structures upon asymmetric observation conditions, were already reported in both scattering ${ }^{29-31}$ and $\mathrm{PLE}^{16}$ experiments, and they have been sometimes referred 
to as extrinsic chirality ${ }^{29,30}$ and pseudochirality. ${ }^{32}$ They are due to the fact that, even for an achiral structure, the overall experimental configuration, including the oblique collection direction and the structure, is geometrically chiral. For achiral structures, the DCP is antisymmetric for reflections across the lines $k_{x}=0$ and $k_{y}=0$. In other words, each emission direction with a preferential circular polarization is compensated by another (specular) direction for which the circular polarization is reversed. Overall, the integrated chirality of the light emitted in the $z$-positive half-space is zero, even though emission into particular directions is preferentially handed. When considering the chiral structures, the DCP shows striking differences. In the center and right columns of Figure 5, we report the DCP for the $D_{+}$(center column) and the $D_{-}$(right column) structures for the same wavelength selection. The color plots show a clear breaking of symmetry between RCP and LCP emission, meaning that the DCP averaged over the back aperture now shows a net bias toward a particular handedness. This handedness is inverted when the geometric chirality is inverted. Let us focus, for example, on the case of $\lambda=720 \mathrm{~nm}$ (Figure $5 \mathrm{~b}, \mathrm{~g}, \mathrm{n}$ ). The $D_{+}$structures (panel g) redirect the LCP light (i.e., blue color) to the same directions as the achiral structures (panel b), but no circularly polarized light is present (i.e., DCP $=0$, white color) at angles into which the achiral structure directs RCP light. The $D_{-}$antennas (panel $\mathrm{n}$ ) behave in exactly the opposite way: they emit RCP light at the same angles as the achiral structure, but the corresponding degree of LCP at the specular emission angles is absent.

From the Fourier images in Figure 4 and Figure 5, we conclude that when reporting a PLE measurement, such as the one by Meinzer ${ }^{17}$ and the ones reported in Figure $3 b-d$, it is first important to report the lens opening angle ( $\mathrm{NA}=0.12$ in Figure $3 \mathrm{~b}-\mathrm{d}$ ), and second, that angular integration tends to obscure handed effects. Indeed, as the PLE features strongly change in angle with wavelength, in a high-NA collection optics scenario where one integrates over (rather than resolves) an angle, no marked PLE features would stand out. The handed PLE asymmetry reported in Figure 4 and by Meinzer et al. ${ }^{17}$ for a cone of angles integrated around the normal direction is hence only very modest compared to the actual angle-resolved PLE asymmetries.

Finally, as the dye used in this experiment is achiral, and the structures that we study are planar, one does not expect net circular polarization when integrating over all solid angles. For the achiral structure, a global vanishing of preferential emission helicity is already evident from the antisymmetry of the DCP. The planar chiral structures, instead, redirect light with a preferential circular polarization to the $z>0$ semispace. In other words, the system formed by the dye and the antennas behaves like an apparent chiral emitter into one-half space. As already pointed out by other authors, ${ }^{17}$ this asymmetry toward one semispace (i.e., $z>0$ ) is compensated by an opposite asymmetry in the other semispace. This interpretation is confirmed by comparing Figure 5 with the measurements shown in Figure $3 \mathrm{~b}-\mathrm{d}$. These show an opposite helicity preference consistent with the fact that light is collected from opposing sample sides. As a caveat, we do note that the air/ glass interface introduces a further symmetry breaking, whereby the above reasoning does not hold above NA $=1$ (not reported in our data).

Comparison to a Theoretical Model. We now compare the results of our experiment with the prediction of a coupled dipole model, ${ }^{27}$ which is described in detail in the Methods section. Each antenna is modeled by an electric dipole located in its center and characterized by an electric polarizability tensor. For a certain external excitation of the system, we calculate the dipole moment acquired by each dipole, and from it, we compute the far-field electromagnetic field. In this way, the polarized angular emission of the sample can be reconstructed and the information about the DCP, for example, can be retrieved. Figure 6 shows the results of this procedure
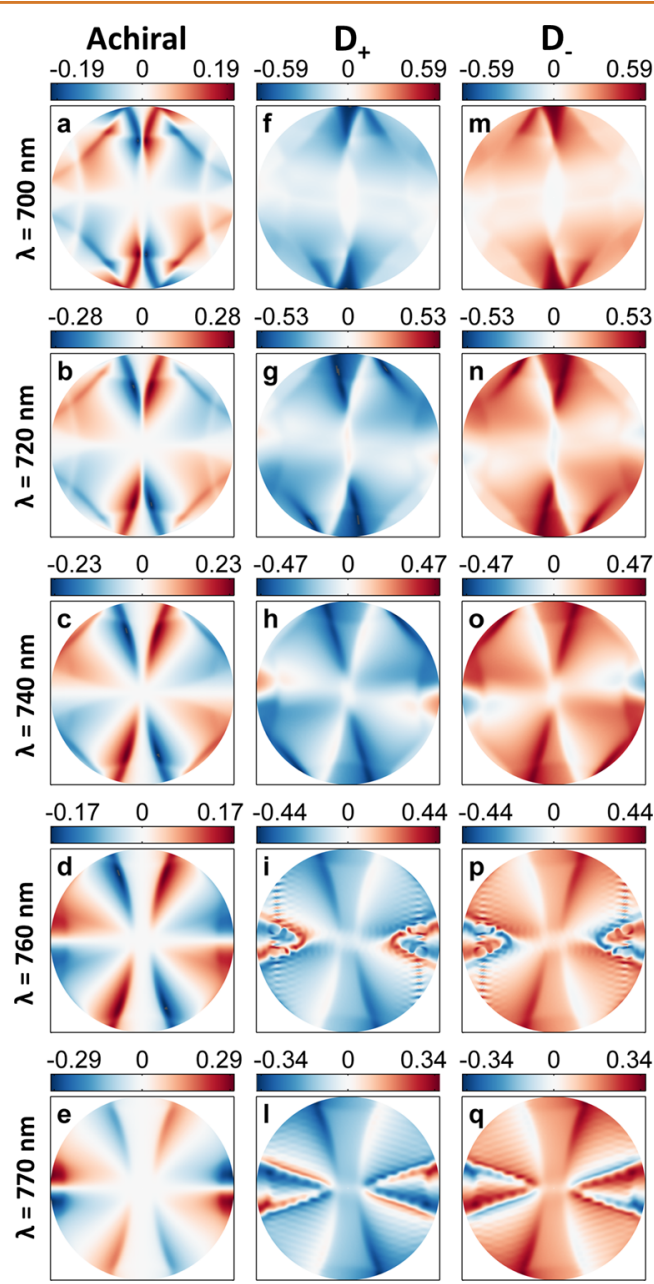

Figure 6. Simulated angle-resolved DCP of the emission from the achiral and chiral structures. The horizontal and vertical axes for each plot are the same as those in Figure $4 a-f$. Each row of the panel corresponds to fixed emission wavelength, ranging from $\mathbf{7 0 0}$ $\mathrm{nm}$ (top row) to $770 \mathrm{~nm}$ (bottom row), as indicated in the label. For each row, the three plots correspond, from left to right, to the emission from the achiral, $D_{+}$, and $D_{-}$structures. The color bar of each plot is indicated on top of it.

for the same set of unit cell chirality and wavelengths used in Figure 5. The calculations reproduce quite well the main results observed in the experiment, that is, a strongly handed response especially near the SLR features, whose handedness is greatly affected by the geometric chirality of the unit cell. Also, the symmetries, indicating null net handedness for the achiral unit cell and net handed emission otherwise, are clearly reproduced. Discrepancies from the measured DCP are also evident, in particular in the regions with $k_{x} \simeq 0.7$ and $k_{y} \simeq 0$, where the DCP has opposite signs (compare Figure 5a and Figure 6a) and/or remarkably different values (e.g., Figure 5e and Figure $6 \mathrm{e})$. We attribute these discrepancies to the inherent limitation 
of the dipole model that inaccurately estimates rod-rod coupling for closely spaced rods. At close spacing, hybridization physics requires the real shape of the fabricated structures to be taken into account. A first pointer that the DCP is indeed highly geometry-dependent is already given by the dipole model: as the amplitudes of the RCP and LCP light depend on a coherent sum of scattered $E_{x}$ and $E_{y}$ fields, varying the amplitude and phase of the polarizability tensor components $\alpha_{x x}$ and $\alpha_{y y}$ quite strongly influences the far-field polarization state. While from a modeling point of view this poses a challenge, from a measurement point of view, this underlines that $k$-space polarimetry can sensitively discriminate between proposed models.

\section{CONCLUSIONS}

We investigated the angular distribution of helicity-dependent photoluminescence enhancement from arrays of planar chiral plasmonic nanostructures embedded in a light-emitting dye layer. Despite the achiral nature of the emitters, this system presents a distinct dissymmetry in the far-field emission between the RCP and LCP light, which is controlled by the geometrical handedness of the array unit cell. While previous works experimentally investigated this effect by sampling photoluminescence orthogonal to the plane of the array (i.e., $k \simeq 0$ ), in this work we found a remarkable and nontrivial distribution of the DCP into the far-field, characterized by angularly narrow areas of high values of the DCP. The angular position of these strong chiral dissymmetries coincides with surface lattice resonances, thus highlighting the important role of diffractive coupling in the light spin-orbit effect. Our findings demonstrate how an array of planar chiral nanostructures can efficiently redirect light from achiral emitters into different narrow directions with distinct circular polarization states. Moreover, these results demonstrate that, when studying the spin-orbit effect in a periodic array of plasmonic nanostructures, $k$-space measurements are an essential tool because sampling only the $k \simeq 0$ portion of the angular spectra cannot reveal the intricate angle-dependent effects.

\section{METHODS}

Sample Fabrication. The sample was fabricated by spin-coating a positive resist (PMMA $950 \mathrm{~A} 4$ ) on a $24 \times 24 \times 0.7 \mathrm{~mm}^{3}$ glass coverslide (Menzel), which was first cleaned with a $\mathrm{H}_{2} \mathrm{O} / \mathrm{H}_{2} \mathrm{O}_{2}$ / $\mathrm{NH}_{4} \mathrm{OH}$ base piranha solution. Square arrays of antennas, with a side of about $300 \mu \mathrm{m}$, were defined through electron beam lithography (30 $\mathrm{keV}$, RAITH150-TWO) with a typical dose of $400 \mu \mathrm{C} / \mathrm{cm}^{2}$. After development with a MIBK/IPA $=1: 3$ solution, we thermally evaporated $2 \mathrm{~nm}$ of chromium followed by $30 \mathrm{~nm}$ of silver at an evaporation rate of $0.5-1 \AA / \mathrm{s}$. Lift-off was performed by keeping the sample in acetone vapor overnight and subsequently immersing it in liquid acetone for $1 \mathrm{~h}$.

In order to deposit a dye-doped layer on top of the fabricated plasmonic structures, a $10 \mathrm{mM}$ solution of cyclopentanone and Rhodamine $800(\mathrm{Rh} 800)$ was mixed with the photoresist SU8 (Microchem SU8-2005) in a 1:1 ratio. To decrease its viscosity and obtain thinner layers, we further diluted $2 \mathrm{~mL}$ of this solution with 8 $\mathrm{mL}$ of cyclopentanone. The resulting solution was spun on the sample at a speed of $4000 \mathrm{rpm}$ for $45 \mathrm{~s}$, resulting in a layer thickness of about $65 \mathrm{~nm}$. The sample was finally baked for $2 \mathrm{~min}$ at $95^{\circ} \mathrm{C}$ to evaporate the cyclopentanone. The Rh800 dye has an absorption maximum at about $680 \mathrm{~nm}$, and its emission maximum at about $720 \mathrm{~nm}$, as shown in Figure 1d.

Setup for $\boldsymbol{k}$-Space Polarimetry. Figure if shows the setup used to perform the $k$-space polarimetry measurements. A linearly polarized supercontinuum laser (Fianium), filtered with an acousto-optical tunable filter (AOTF, Crystal Technologies) and a band-pass filter $(620-10 \mathrm{~nm})$, is focused on the sample by an achromatic lens. The power of the laser, measured before the lens, is about $16 \mu \mathrm{W}$. The fluorescence is collected from the other side of the sample with a $60 \mathrm{X}$ objective (NA $=0.7$, Nikon CFI Plan Fluor) and separated from the excitation light by a $650 \mathrm{~nm}$ long-pass filter. The desired state of polarization is selected by a polarimeter, composed of a QWP and a linear polarizer. A subsequent spatial filter, composed of a 1:1 telescope $\left(f_{\text {telescope }}=50 \mathrm{~mm}\right)$ and a $400 \mu \mathrm{m}$ pinhole, ensures that only the signal coming from an area of about $25 \mu \mathrm{m}$ on the sample is collected, so that the array edges for our finite sample fields do not affect the measurement. The Fourier (or Bertrand) lens $\left(f_{\text {Fourier }}=200\right.$ $\mathrm{mm}$ ) is mounted on a flippable stage, allowing either the real space or the back focal plane of the objective to be imaged. Finally, a tube lens $\left(f_{\text {tube }}=200 \mathrm{~mm}\right)$ focuses the light on a silicon CCD camera (Photometrics CoolSnap EZ). The exposure time of the camera was $60 \mathrm{~s}$ for all measurements. A set of narrow ( $\mathrm{fwhm}=10 \mathrm{~nm}$ ) band-pass filters (in the range of $700-790 \mathrm{~nm}$ ) has been used in front of the camera to acquire quasi-monochromatic images.

In this configuration, the signal collected by the camera is directly proportional to the intensity of the polarization state selected by the polarimeter. In more complex systems, in which other optical components are present between the sample and the polarizer, the polarization state of light is transformed according to the Mueller matrix of each element. This matrix can be calculated from preliminary calibration measurements ${ }^{22,23}$ and therefore used to correct for the polarization conversion effects due to each optical element.

Setup for Low-NA Transmission and PLE. In order to both benchmark our results against those of Meinzer et al. ${ }^{17}$ and to show the role of the SLR, we measured the PLE and transmittance (Figure 3 ) in a low-NA normal-incidence configuration. We used a simplified version of the setup described in Figure 1f, in which the collection objective is replaced by a low-NA achromatic lens $(\mathrm{NA}=0.12)$ and the Fourier lens is removed, as shown in Figure $7 \mathrm{a}, \mathrm{b}$. Instead of imaging the emission on the camera CCD, it is focused on a fiber and directed to the entrance slit of a spectrometer. For the transmittance measurements (Figure $7 \mathrm{~b}$ ), we illuminated the sample with linearly polarized white light from a fiber-coupled halogen lamp without any polarization analysis on the transmitted light (i.e., the polarimeter is removed).

Polarimetry Principle. Each set of angle-resolved Stokes parameters was retrieved based on six consecutive Fourier images, according to

$$
\begin{aligned}
& S_{0}=I_{\mathrm{V}}+I_{\mathrm{H}} \\
& S_{1}=I_{\mathrm{V}}-I_{\mathrm{H}} \\
& S_{2}=I_{+}-I_{-} \\
& S_{3}=I_{\mathrm{R}}-I_{\mathrm{L}}
\end{aligned}
$$

where $I_{\mathrm{H}}, I_{\mathrm{V}}, I_{+}$, and $I_{-}$denote the intensities of the linear horizontal, vertical, diagonal, and antidiagonal polarized components of the emission, while $I_{\mathrm{L}}$ and $I_{\mathrm{R}}$ denote the intensities of the LCP and RCP components, respectively. Once the Stokes parameters have been obtained through the measurements of the six intensities mentioned above, all other quantities related to polarization can be calculated. In particular, the degrees of polarization DP, linear polarization DLP, and circular DCP can be derived from the Stokes parameters ${ }^{33}$

$$
\begin{aligned}
& \mathrm{DP}=\frac{\sqrt{S_{1}^{2}+S_{2}^{2}+S_{3}^{2}}}{S_{0}} \\
& \mathrm{DLP}=\frac{\sqrt{S_{1}^{2}+S_{2}^{2}}}{S_{0}} \\
& \mathrm{DCP}=\frac{S_{3}}{S_{0}}
\end{aligned}
$$




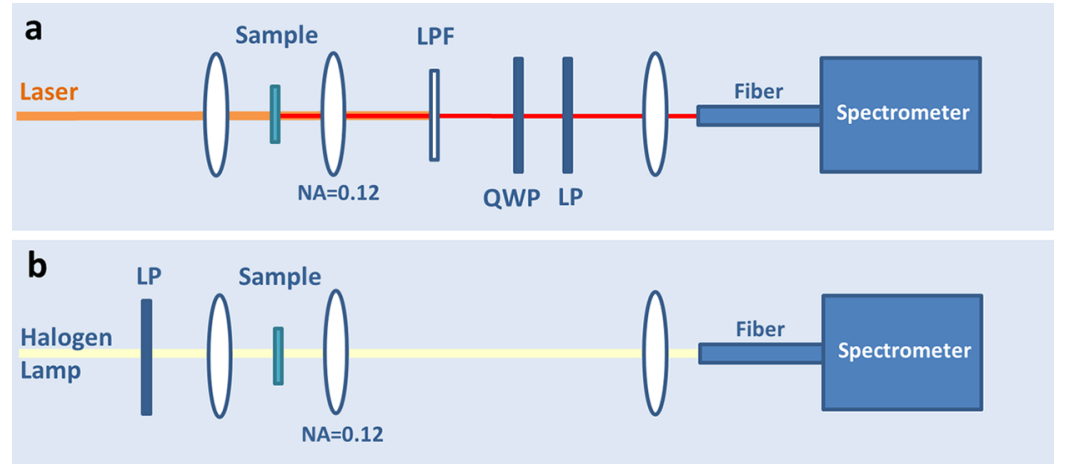

Figure 7. Scheme of the setups used for (a) PLE and (b) transmittance measurements.

These quantities describe the ratio of polarized, linearly polarized, and circularly polarized light to total emission. For the DCP definition, the absolute value of $S_{3}$ is commonly used. With our definition, the DCP is a signed quantity which can vary from -1 (light is completely LCP) to +1 (light is completely RCP).

Coupled Dipole Model. Each antenna is modeled by an electric dipole located in its center and characterized by an electric polarizability tensor $\alpha$. If the system is excited by a monochromatic external field $\mathbf{E}_{\text {inc }}$ at frequency $\omega$, the dipole moment $\mathbf{p}_{i}$ acquired by the ith dipole is

$$
\mathbf{p}_{i}=\alpha(\omega) \mathbf{E}_{\mathrm{inc}, i}+\alpha(\omega) \sum_{j \neq i} \mu \omega^{2} G\left(\mathbf{r}_{i}, \mathbf{r}_{j}, \omega\right) \mathbf{p}_{j}
$$

where $\mathbf{E}_{\text {inc,i }}$ denotes the incident field at the position of the $i$ th dipole, $\mu$ is the magnetic permeability, and $G\left(\mathbf{r}_{i}, \mathbf{r}_{j}, \omega\right)$ is the Green tensor of the system, which quantifies the propagation of the electromagnetic field from a dipole at position $\mathbf{r}_{j}$ to position $\mathbf{r}_{i}$. The sum is performed over all $N$ dipoles. The problem can be easily cast in a system of $3 N$ algebraic equations and solved with numerical methods to obtain the dipole moments $\mathbf{p}_{1}, \mathbf{p}_{2}, \ldots \mathbf{p}_{N}$. Once the dipole moments are known, the far-field electromagnetic field can be obtained by using the appropriate asymptotic far-field Green function $G_{\mathrm{FF}}{ }^{27,34}$

$$
\mathbf{E}_{\text {far field }}(\theta, \phi)=\sum_{i=1}^{N} \mu \omega^{2} G_{\mathrm{FF}}\left(\theta, \phi, \mathbf{r}_{i}, \omega\right) \mathbf{p}_{i}
$$

In this way, the polarized angular emission of the sample can be reconstructed and the information about the DCP, for example, can be retrieved. Our system is described by a three-layer structure with refractive indexes $n_{\text {glass }}=1.52, n_{\mathrm{SU} 8}=1.58$, and $n_{\text {air }}=1$. The Green tensors of such stratified structures are calculated numerically starting from analytical formulas. ${ }^{27}$ We simulated arrays with $61 \times 61$ unit cells (i.e., 7442 single dipoles) and with different chirality of the unit cell. The polarizability tensor of a single rod is calculated by adding two correction terms to the static polarizability ${ }^{35,36}$ of a silver rod to take in account the depolarization induced by the accumulated surface charges and the radiation damping. ${ }^{36}$ The static polarizability and the depolarization factor depend on the rod dimensions, while the radiation damping depends on the environment in which the dipole is placed (i.e., the three-layer stack, in our case). For the permittivity of the silver, we assumed a Drude model, $\epsilon(\omega)=1-\omega_{\mathrm{p}}^{2} /\left(\omega^{2}-i \omega \gamma\right)$, with $\omega_{\mathrm{p}}=2 \pi \times 2.321 \times 10^{15} \mathrm{~Hz}$ and $\gamma=2 \pi \times 5.513 \times 10^{12} \mathrm{~Hz}$.

In order to simulate the excitation of the nanoantennas by nearby fluorescent emitters, we used, as driving field, several dipolar sources with different polarization and in different positions inside the SU8 layer. The far-field electric field is calculated separately for each source. Afterward, the total far-field polarized intensities are obtained by summing incoherently the contribution of each source. Finally, the DCP is calculated according to eq $2 \mathrm{c}$. Moreover, when using eq 4 , a Gaussian spatial filter is applied in order to smoothly remove the contribution from dipoles located close to the array's edge.

\section{ASSOCIATED CONTENT}

\section{Supporting Information}

The Supporting Information is available free of charge on the ACS Publications website at DOI: 10.1021/acsnano.5b07231.

Additional numerical calculation of the sample transmittance, near-field patterns for localized and lattice resonances (PDF)

\section{AUTHOR INFORMATION}

\section{Corresponding Author}

*E-mail: m.cotrufo@tue.nl.

\section{Notes}

The authors declare no competing financial interest.

\section{ACKNOWLEDGMENTS}

We thank H. Schokker for assistance with sample fabrication, C. Guo and L. Langguth for providing the scripts for the coupled dipole model calculations, and A. Fiore for fruitful discussions. The work is part of the research programme of the Foundation for Fundamental Research on Matter (FOM), which is financially supported by The Netherlands Organisation for Scientific Research (NWO). The work of C.I.O. and A.F.K. is supported by NanoNextNL, a micro- and nanotechnology consortium of the Government of The Netherlands and 130 partners. A.F.K. gratefully acknowledges an NWO-Vidi grant for financial support.

\section{REFERENCES}

(1) Novotny, L.; van Hulst, N. Antennas for light. Nat. Photonics 2011, 5, 83-90.

(2) Muskens, O.; Giannini, V.; Sanchez-Gil, J.; Gómez Rivas, J. Strong Enhancement of the Radiative Decay Rate of Emitters by Single Plasmonic Nanoantennas. Nano Lett. 2007, 7, 2871-2875.

(3) Kinkhabwala, A.; Yu, Z.; Fan, S.; Avlasevich, Y.; Müllen, K.; Moerner, W. Large Single-Molecule Fluorescence Enhancements Produced by a Bowtie Nanoantenna. Nat. Photonics 2009, 3, 654-657.

(4) Akselrod, G. M.; Argyropoulos, C.; Hoang, T. B.; Ciraci, C.; Fang, C.; Huang, J.; Smith, D. R.; Mikkelsen, M. H. Probing the Mechanisms of Large Purcell Enhancement in Plasmonic Nanoantennas. Nat. Photonics 2014, 8, 835-840.

(5) Valev, V. K.; Baumberg, J. J.; Sibilia, C.; Verbiest, T. Chirality and Chiroptical Effects in Plasmonic Nanostructures: Fundamentals, Recent progress, and Outlook. Adv. Mater. 2013, 25, 2517-2534.

(6) Kuwata-Gonokami, M.; Saito, N.; Ino, Y.; Kauranen, M.; Jefimovs, K.; Vallius, T.; Turunen, J.; Svirko, Y. Giant Optical Activity in Quasi-Two-Dimensional Planar Nanostructures. Phys. Rev. Lett. 2005, 95, 227401. 
(7) Gorodetski, Y.; Shitrit, N.; Bretner, I.; Kleiner, V.; Hasman, E. Observation of Optical Spin Symmetry Breaking in Nanoapertures. Nano Lett. 2009, 9, 3016-3019.

(8) Kuzyk, A.; Schreiber, R.; Fan, Z.; Pardatscher, G.; Roller, E.-M.; Högele, A.; Simmel, F. C.; Govorov, A. O.; Liedl, T. DNA-Based SelfAssembly of Chiral Plasmonic Nanostructures with Tailored Optical Response. Nature 2012, 483, 311-314.

(9) Song, C.; Blaber, M. G.; Zhao, G.; Zhang, P.; Fry, H. C.; Schatz, G. C.; Rosi, N. L. Tailorable Plasmonic Circular Dichroism Properties of Helical Nanoparticle Superstructures. Nano Lett. 2013, 13, 32563261.

(10) Decker, M.; Ruther, M.; Kriegler, C.; Zhou, J.; Soukoulis, C.; Linden, S.; Wegener, M. Strong Optical Activity from Twisted-Cross Photonic Metamaterials. Opt. Lett. 2009, 34, 2501-2503.

(11) Gorkunov, M.; Ezhov, A.; Artemov, V.; Rogov, O.; Yudin, S. Extreme Optical Activity and Circular Dichroism of Chiral Metal Hole Arrays. Appl. Phys. Lett. 2014, 104, 221102.

(12) Eftekhari, F.; Davis, T. J. Strong Chiral Optical Response from Planar Arrays of Subwavelength Metallic Structures Supporting Surface Plasmon Resonances. Phys. Rev. B: Condens. Matter Mater. Phys. 2012, $86,075428$.

(13) Fan, Z.; Govorov, A. O. Plasmonic Circular Dichroism of Chiral Metal Nanoparticle Assemblies. Nano Lett. 2010, 10, 2580-2587.

(14) Schäferling, M.; Dregely, D.; Hentschel, M.; Giessen, H. Tailoring Enhanced Optical Chirality: Design Principles for Chiral Plasmonic Nanostructures. Phys. Rev. X 2012, 2, 031010.

(15) Hendry, E.; Carpy, T.; Johnston, J.; Popland, M.; Mikhaylovskiy, R. V.; Lapthorn, A. J.; Kelly, S. M.; Barron, L. D.; Gadegaard, N.; Kadodwala, M. Ultrasensitive Detection and Characterization of Biomolecules Using Superchiral Fields. Nat. Nanotechnol. 2010, 5, 783-787.

(16) Kruk, S. S.; Decker, M.; Staude, I.; Schlecht, S.; Greppmair, M.; Neshev, D. N.; Kivshar, Y. S. Spin-Polarized Photon Emission by Resonant Multipolar Nanoantennas. ACS Photonics 2014, 1, 12181223.

(17) Meinzer, N.; Hendry, E.; Barnes, W. L. Probing the Chiral Nature of Electromagnetic Fields Surrounding Plasmonic Nanostructures. Phys. Rev. B: Condens. Matter Mater. Phys. 2013, 88, 041407.

(18) Vecchi, G.; Giannini, V.; Gomez Rivas, J. Surface Modes in Plasmonic Crystals Induced by Diffractive Coupling of Nanoantennas. Phys. Rev. B: Condens. Matter Mater. Phys. 2009, 80, 201401.

(19) Rodriguez, S.; Schaafsma, M.; Berrier, A.; Gomez Rivas, J. Collective Resonances in Plasmonic Crystals: Size Matters. Phys. B 2012, 407, 4081-4085.

(20) Lozano, G.; Louwers, D. J.; Rodríguez, S. R.; Murai, S.; Jansen, O. T.; Verschuuren, M. A.; Gomez Rivas, J. Plasmonics for Solid-State Lighting: Enhanced Excitation and Directional Emission of Highly Efficient Light Sources. Light: Sci. Appl. 2013, 2, e66.

(21) Lozano, G.; Grzela, G.; Verschuuren, M. A.; Ramezani, M.; Rivas, J. G. Tailor-Made Directional Emission in Nanoimprinted Plasmonic-Based Light-emitting Devices. Nanoscale 2014, 6, 92239229.

(22) Osorio, C. I.; Mohtashami, A.; Koenderink, A. K-space Polarimetry of Bullseye Plasmon Antennas. Sci. Rep. 2015, 5, 9966.

(23) Mohtashami, A.; Osorio, C. I.; Koenderink, A. F. AngleResolved Polarimetry Measurements of Antenna-Mediated Fluorescence. arXiv:1506.00140 2015.

(24) Fedotov, V.; Mladyonov, P.; Prosvirnin, S.; Rogacheva, A.; Chen, Y.; Zheludev, N. Asymmetric Propagation of Electromagnetic Waves through a Planar Chiral Structure. Phys. Rev. Lett. 2006, 97, 167401.

(25) Lumerical Solutions, Inc. http://www.lumerical.com/tcadproducts $/ \mathrm{fdtd} /$.

(26) Murai, S.; Verschuuren, M.; Lozano, G.; Pirruccio, G.; Rodriguez, S.; Rivas, J. G. Hybrid Plasmonic-Photonic Modes in Diffractive Arrays of Nanoparticles Coupled to Light-Emitting Optical Waveguides. Opt. Express 2013, 21, 4250-4262.

(27) Novotny, L.; Hecht, B. Principles of Nano-Optics; Cambridge University Press: New York, 2006.
(28) Langguth, L.; Schokker, A.; Guo, K.; Koenderink, A. Plasmonic Phase-Gradient Metasurface for Spontaneous Emission Control. Phys. Rev. B: Condens. Matter Mater. Phys. 2015, 92, 205401.

(29) Plum, E.; Fedotov, V.; Zheludev, N. Extrinsic Electromagnetic Chirality in Metamaterials. J. Opt. A: Pure Appl. Opt. 2009, 11, 074009.

(30) Lu, X.; Wu, J.; Zhu, Q.; Zhao, J.; Wang, Q.; Zhan, L.; Ni, W. Circular Dichroism from Single Plasmonic Nanostructures with Extrinsic Chirality. Nanoscale 2014, 6, 14244-14253.

(31) Sersic, I.; van de Haar, M. A.; Bernal Arango, F.; Koenderink, A. F. Ubiquity of Optical Activity in Planar Metamaterial Scatterers. Phys. Rev. Lett. 2012, 108, 223903.

(32) Lindell, I. V.; Sihvola, A. H.; Tretyakov, S. A.; Viitanen, A. J. Electromagnetic Waves in Chiral and Bi-isotropic Media; Artech House: Norwood, MA, 1994.

(33) Goldstein, D. H. Polarized Light; CRC Press: Boca Raton, FL, 2010.

(34) Lukosz, W. Light Emission by Magnetic and Electric Dipoles Close to a Plane Dielectric Interface. III. Radiation Patters of Dipoles with Arbitrary Orientation. J. Opt. Soc. Am. 1979, 69, 1495-1503.

(35) Maier, S. A. Plasmonics: Fundamentals and Applications; Springer Science \& Business Media: Berlin, 2007.

(36) Hofmann, H. F.; Kosako, T.; Kadoya, Y. Design Parameters for a Nano-Optical Yagi-Uda Antenna. New J. Phys. 2007, 9, 217. 